\title{
Investigations into the Chemical Structure Based Selectivity of the Microfabricated Nitrogen-Phosphorus Detector
}

\author{
Terisse A. Brocato, Ryan F. Hess, Matthew Moorman, Robert J. Simonson
}

\begin{abstract}
Nitrogen and phosphorus atoms are constituents of some of the most toxic chemical vapors. Nitrogen-phosphorus gas chromatograph detectors (NPDs) rely on selective ionization of such compounds using ionization temperatures typically greater than $600^{\circ} \mathrm{C}$. NPDs have previously been reported to be $7^{*} 10^{4} x$ and $10^{5} x$ more sensitive for nitrogen and phosphorus, respectively, than for carbon. Presented here is an investigation of the structure-based selectivity of a microfabricated nitrogen-phosphorus detector ( $\mu N P D)$. The $\mu$ NPD presented here is smaller than a dime and can be placed in a system that is $1 / 100^{\text {th }}$ the size of a commercial NPD. Comparison of responses of such devices to homologous anilines ( $p$-methoxyaniline, $p$-fluoroaniline, and aniline) revealed that detection selectivity, determined by the ratio of $\mu$ NPD to nonselective flame ionization detector (FID) peak areas, is correlated with acid disassociation $\mathrm{pK}_{\mathrm{a}}$ values for the respective analine. Selectivity was determined to be greatest for $p$-methoxyaniline, followed by $p$-fluoroaniline, with aniline having the smallest response. The limit of detection for a nitrogen containing chemical, p-methoxyaniline, using the $\mu$ NPD was determined to be $0.29 \mathrm{ng}$ compared to $59 \mathrm{ng}$ for a carbon chemical containing no nitrogen or phosphorus, 1,3,5-trimethybenzene. The $\mu$ NPD presented here has increased detection for nitrogen and phosphorus compared to the FID and with a slight increase in detection of carbon compounds compared to commercial NPD's sensitivity to nitrogen and carbon.
\end{abstract}

Key words: Acid disassociation constant $\left(\mathrm{pK}_{\mathrm{a}}\right)$; Aniline; Nitrogen; Phosphorus; Chemical detector

\section{Introduction}

A nitrogen phosphorus gas chromatograph detector (NPD) is a thermionic detector first created by Kolb and Bischoff, and commercialized by Patterson [1]. Heating of a solid surface, typically of a Cs- or $\mathrm{Rb}$-doped silicate ceramic bead, causes an increase in current emission in the presence of electronegative samples [2]. This increase in current emission allows for detection, seen as a change in current emission over a period of time. The NPD is a particular thermionic detector which is selective for molecules containing nitrogen and phosphorus [3]. Two broad classes of thermally induced ionization mechanisms have been proposed in the literature: a gas phase ionization theory proposed by Kolb and Bischoff, and surface ionization theory proposed by Patterson [3, 4]. Attempts to detect gas phase ionization intermediates by laser-induced fluorescence have been unsuccessful, lending some indirect

This study was supported by Sandia National Laboratories. Sandia National Laboratories is a multi-program laboratory managed and operated by Sandia Corporation, a wholly owned subsidiary of Lockheed Martin Corporation, for the U.S. Department of Energy's National Nuclear Security Administration under contract DE-AC04-94AL85000. 
support for surface-mediated rather than gas-phase ionization. However, details of the selective ionization mechanism(s) involved are still not understood.

Commercial NPDs typically exhibit sensitivities of $7 * 10^{4} x$ and $10^{5} x$ for nitrogen and phosphorus heteroatoms, respectively, as compared to carbon[2]. This specificity is extremely advantageous for detection of trace amounts of $\mathrm{N}$ - and $\mathrm{P}$-containing toxic chemicals such as toxic industrial chemicals, nitrogen-containing explosives, or pesticides, particularly in complex sample matrices containing significant hydrocarbon background concentrations.

$\mu N P D$ chemical detection uses the detection of electron emission based on the chemical exposed to the device. Creating a sensitive detector for $\mathrm{N}$ and $\mathrm{P}$ required us to look into factors that alter thermionic electron emission current. Thermionic electron emission current from a hot surface follows the Richardson-Dushman equation as seen in Equation (1)[5].

$J=A T^{2} \exp [-\phi / k T]$

Where $\mathrm{J}$ is the current density, $\mathrm{A}$ is the Richardson's constant, $\mathrm{T}$ is temperature, $\mathrm{k}$ is Boltzmann's constant, and $\phi$ is the work function of the surface.

Although the relation between free electron emission described by Equation (1) and selective molecular ionization is indirect, the response of the NPD is a strong function of substrate temperature. In the experiments reported here the $\mu N P D$ is run at temperatures in the range of $400-550^{\circ} \mathrm{C}$, somewhat lower than typical operating temperatures for macroscopic NPD operation. This operating temperature range is a compromise between increased ionization signal and operating lifetime of the relatively thin $\mu N P D$ emitter coatings. These coatings consist of emitters that were spray coated with porous silica containing alkali metals. Rubidium ( $\mathrm{Rb}$ ) and cesium ( $\mathrm{Cs}$ ) are commonly used for the alkali metal in the spray coating in order to lower the work function of the heated surface. A lower work function allows for increased thermionic emission of electrons at a lower temperature [2], due to the exponential term in Equation (1).

Typically NPD systems including a benchtop gas chromatograph with an NPD cost approximately $\$ 15,000-\$ 40,000$ and are $\sim 2 \mathrm{ft}^{3}$ or larger [6]. The gas cylinders required to run the gas chromatograph also adding considerably to system size. Detectors with high sensitivity come at the price of large size, increased detection time, and the need for a skilled operator. There are currently commercially available benchtop NPDs which have detection limits of $10 \mathrm{ppb} N$, with $\sim 10 x$ better detection for $P$ [6]. The limit of detection for $\mathrm{p}$-methoxyaniline $\mu$ NPD corresponds to $64.9 \mathrm{ppm} \mathrm{N}$. This indicates that our portable, handheld $\mu N P D$ has a somewhat reduced detection ability versus the commercial NPD, but at $1 / 100^{\text {th }}$ the size. The $\mu$ NPD was designed with the idea that it would be most beneficial to people working in the field.Figure 1 shows a $\mu$ NPD device which is much smaller than a dime, $6.7 \times 4.6 \mathrm{~mm}$, and can be placed in handheld chemical detection systems.

In what follows, we describe our microfabricated nitrogen phosphorus detector ( $\mu N P D)$ and present results for detection of a series of homologous amines as well as the comparison to the common flame ionization detector used with gas chromatographs. Specifically, substituted anilines 
were tested to determine variations in selectivity due to the addition of functional groups to the aniline ring. This research was performed in order to seek insight into the thermal ionization mechanism(s) of the $\mu$ NPD by attempting to vary the electron affinity of the functional groups on the aniline. An improved understanding of detection selectivity can help to understand detection mechanisms, with the ultimate goal of improving detection limits for chemicals that are toxic in trace amounts.

\section{Materials and Methods}

\section{1. $\mu N P D$ Design}

The $\mu$ NPD device is a Pt-ZnO microhotplate which contains Pt as a base with a ZnO adhesion layer. We at Sandia National Laboratories created a material system for use as a Micro Electro Machined System (MEMs) thin film heater. This is the first report of that material system and its performance in a sensor system. The $\mathrm{ZnO}$ material forms a superior adhesion layer that encourages the growth and stabilization of grain boundaries within the Pt metal. This prevents the delamination of the thin film, but it also greatly increases the resistance of the Pt to agglomeration, which is a common failure mechanism of thin films at high temperature. The ZnO layer was deposited via sputter deposition and patterned using photoresist lift-off.

\subsection{Cesium Hydroxide/Rubidium Hydroxide Sol Gel}

In a fume hood, cesium hydroxide monohydrate (50 wt\% solution) or rubidium hydroxide (50 wt\% solution) was mixed with water and tetraethyl orthosilicate (TEOS). A TEOS:water ratio of 1:5, and a cesium (or rubidium):silicon ratio of 3:1 was used to make the sol-gel mixture. The sol-gel was then stirred for about three hours until the two phase solution (the organic TEOS on top of the aqueous metal ion solution) became monophasic. Once monophasic, the solution was loaded into a syringe, placed in an automated syringe pump, and spray coated. The region coated is on the backside of the $\mu$ NPD devices, in the material holding cup. The $\mu$ NPD device was coated on the backside in order to prevent delamination of the wiring as a result of sol-gel $\mathrm{ZnO}$ interactions.

\subsection{Spray Coating}

The $\mu$ NPD sensor surface was prepared by heating it to approximately $480^{\circ} \mathrm{C}$ for 10 minutes prior to spray coating. This allowed annealing of the $\mathrm{Pt}$ to the $\mathrm{ZnO}$ prior to coating and heating.Spray coating $\mu$ NPD devices was conducted using an ultrasonic spray coater produced by Sono-Tek Corporation. The ultrasonic nozzle power was set at $2.5 \mathrm{~W}$ at room temperature. Devices were coated using a flow rate of $10 \mathrm{~mL} / \mathrm{hr}$ and 15 passes. After being coated, the devices were placed in a tube furnace and heated to $350^{\circ} \mathrm{C}$ under flowing nitrogen gas for two hours. The ramp up and down rate was set at $60^{\circ} \mathrm{C} / \mathrm{hr}$. This heating set is thought to increase the rate of the cross-linking reaction and to burn off excess solvent. After the devices are removed from the furnace they are ready for use in the NPD fixture. Devices were then heated to $450^{\circ} \mathrm{C}$ in the $\mu$ NPD fixture for approximately 20 minutes prior to taking data in order to remove any excess solvent remaining after being in the furnace. 


\section{4. $\mu$ NPD Testing Fixture}

A fixture, composed primarily of stainless steel with Macor $^{\circledR}$ (machinable glass ceramic) inserts, the white pieces in Figure 2, was developed to provide a testing interface for the coated $\mu$ NPD device[7]. The fixture, shown in Figure 2, integrates all of the electrical and gas flow connections into a single setup, and features coaxial line connections to the collector electrodes and pogo pin connections to the $\mu$ NPD device. The fixture features heavy stainless steel walls to provide excellent electrical shielding of the device and the collector electrodes, as well as mechanical robustness against the high thermal loads from prolonged $\mu$ NPD testing. The total stainless steel dimensions are roughly $1.5^{\prime \prime} \times 2$ ". Macor ${ }^{\circledR}$ inserts were used to hold the NPD device and the electrical connections, in order to keep them from shorting to the metal walls. In addition, excess air volume is minimized by the Macor ${ }^{\circledR}$ inserts. The design features electrical connections to the $\mu$ NPD that position the backside of the $\mu$ NPD device in the gas flow channel, and this allows material deposition into the backside cup coated with an alkali hydroxide. Figure 3 shows an exploded image of the fixture,electrode connections, and how the device fits in with the sample gas flow.

\subsection{Setup in Laboratory}

A test run begins with the sample, a mixture of each analyte in acetonitrile, being injected into an Agilent 6890 gas chromatograph. Analytes are separated using the gas chromatograph (GC) using $\mathrm{N}_{2}$ as a carrier and makeup gas. The GC conditions for the experiments are shown below. Each analyte flows though the $\mu$ NPD and a peak is obtained for the $\mu$ NPD, then each analyte flows through a flame ionization detector (FID) and a peak is obtained for the FID. The $\mu$ NPD data is recorded using a program developed using LabVIEW to record the $\mu$ NPD data which was taken using a Keithley Model 6514 Electrometer, see Figure 3 for depiction.FID data is recorded using Agilent ChemStation B.02.01 SR1. The $\mu$ NPD bias conditions are as follows: $20 \mathrm{~V}$ collector bias, $20 \mathrm{~V}$ microhotplate offset bias, $8.82-12 \mathrm{~V}$ microhotplate bias, see Figure 3 to view connections.A microhotplate bias of $8.82-12 \mathrm{~V}$ results in a $\mu$ NPDtemperature of $340-465^{\circ} \mathrm{C}$.

GC conditions for the aniline series of experiments (aniline, p-methoxyaniline, and p-fluoroaniline) are as follows: RTX-1 column (9.3m, .53-mmID, 1.5-um film thickness; Restek), 0.2 ul injection, $250^{\circ} \mathrm{C}$ inlet, $5 \mathrm{psiN}_{2}, 115 \mathrm{~mL} / \mathrm{min}$ total flow, $5: 1$ split ratio, $94.4 \mathrm{~mL} / \mathrm{min}$ split flow, oven- $100^{\circ} \mathrm{C}$, FID- $280^{\circ} \mathrm{C}$, $40 \mathrm{~mL} / \mathrm{minH}_{2}$ flow, $400 \mathrm{~mL} / \mathrm{min}$ air flow. GC conditions for the limit of detection set of data were: RTX-1 column (15m, .25-mmID, 1-um film thickness; Restek). Injector- 4ul headspace injections, inlet- $250^{\circ} \mathrm{C}$ inlet, $45 \mathrm{psiN}_{2}, 68.9 \mathrm{~mL} / \mathrm{min}$ total flow, $6: 1$ split ratio, $55.9 \mathrm{~mL} / \mathrm{min}$ split flow, oven- $100^{\circ} \mathrm{C}$, FID- $280^{\circ} \mathrm{C}$, $35 \mathrm{~mL} / \mathrm{minH}_{2}$ flow, $350 \mathrm{~mL} / \mathrm{min}$ air flow. $X$ volume headspace injections of either $\mathrm{p}$-methoxyaniline or 1,3,5-trimethylbenzene are taken at various temperatures which alters mass injected into gas chromatograph and detector.

\subsection{Determining Selectivity and Limit of Detection of Analytes}

Three analytes were used for determining the selectivity of the $\mu$ NPD: p-fluoroaniline, p-methoxyaniline, and aniline. Each analyte was individually tested at a concentration of $0.1 \mathrm{M}$ in acetonitrile using the 
laboratory setup described previously. These analytes were tested at seven temperatures in the range of $340-465^{\circ} \mathrm{C}$.

Analyte peak area was calculated using OriginPro 9.0 software's peak analyzer function, with endpoints weighted to determine best peak baseline. Peak analysis was performed based on each analyte's elution time at each GC condition.

The limit of detection (LOD) for the $\mu$ NPD and FID was calculated using using Equations (2) \& (3), the method detection limit (MDL) at 99\% confidence and 7 degrees of freedom[8, 9].

$M D L=s * t_{(n-1,1-\alpha=0.99)}=s * t_{(7,0.99)}$

Where $s$ is the standard deviation, $t$ is the student's $t$ value, $1-\alpha$ is the confidence level 0.99 , and $\alpha$ is the level of significance 0.01 .

$L O D=M D L * m / A$

Where $m$ is the mass injected and $A$ is the average peak area.

\subsection{Temperature Calculation of NPD}

For each run, the temperature of the $\mu$ NPD was calculated using a temperature coefficient of resistance (TCR) determined for the device tested up to temperatures of $155^{\circ} \mathrm{C}$. Linear TCR values for the $\mu$ NPD devices allow for extrapolation of temperature values to determine the temperature for each test run.

\section{Results and Discussion}

$\underline{\text { Use of the } \mu N P D \text { in a Portable Chemical Detection System }}$

Using the $\mu$ NPDin a portable chemical detection system would require a system optimized specifically for the $\mu$ NPD. This system would contain a preconcentrator to concentrate a sample, a small GC in order to separate analytes, in addition to a $N$ NPD and a computer to record and analyze data. The data presented here was taken using a benchtop GC, with a gas capillary column that is $9.3 \mathrm{~m}$. This system was put together to test the $\mu$ NPD and determine its capabilities. With this setup the time to get complete elution of an analyte took under 5 minutes for all analytes. In the field a quicker response time will be needed. Use of a shorter capillary column in the portable chemical detection system will minimize the response time, optimally to a matter of seconds. In addition, GCxGC can be used to maximize analyte separation using a shorter GC column.

Using the setup described in the methods section, a newly coated $\mu$ NPDrequires 2 minutes for warmup and 20 minutes for stabilization. Reheating a $\mu$ NPDonly requires stabilization of 2 minutes. A more ideal fixture setup in a portable system would allow for a temperature ramp right before a test run, while a sample is being preconcentrated. Inbetween runs the $\mu$ NPD could be maintained at a lower temperature for longer time periods when not in use. 
Selectivity, Limit of Detection (LOD), and Suggested Mechanism of the $\mu N P D$

Using molecular orbital theory to analyze the structure of anilines, we can predict the electron density in the anilines. In particular we are interested in looking at hybridization of the nitrogen atom, which we found is correlated to the $\mu$ NPD's high sensitivity to $\mathrm{N}$ and $\mathrm{P}$ compounds. In previous experiments pyridine, which contains a sp ${ }^{2}$ hybridized nitrogen atom (nitrogen bound to 2 other atoms and a lone electron pair), was shown to have a much smaller peak area than molecules which contain a $\mathrm{sp}^{3}$ hybridized nitrogen atom (nitrogen bound to 3 other atoms and a lone electron pair), for example piperidine and aniline. $\mathrm{Asp}^{2}$ hybrid orbital has unshared electrons which are more tightly held compared to a $\mathrm{sp}^{3}$ hybrid orbital [3]. Due to this effect, this appears to be a reasonable hypothesis as to why there is a lower current emission for pyridine than for aniline.

In order to further investigate the effect of electron donating properties on $\mu$ NPD detection a set of three anilines were tested:aniline, $\mathrm{p}$-fluoroaniline, and $\mathrm{p}$-methoxyaniline. These analytes were chosen due to their similarity in structure surrounding the $\mathrm{N}$ but differing electron density for the $\mathrm{N}$. Fluorine is an electron withdrawing group which withdraws electrons from the aromatic ring of aniline. Methoxy is an electron donating group which donates electrons to the aromatic ring of aniline. The structures of these anilines are shown in Figure 4.

Results of relative selectivity between the analogous amines and their structures are shown in Figure 4. P-methoxyaniline was shown to have the largest peak area ( $\left.\mathrm{pA}^{*} \mathrm{~s}\right)$, followed by $\mathrm{p}$-fluoroaniline, with aniline having the smallest peak area. Relative selectivity, the ratio of peak areas of $\mu$ NPD: FID (microfabricated nitrogen phosphorus detector: flame ionization detector) of aniline, $p$-fluoroaniline, and p-methoxyaniline at various operational temperatures are compared in Figure 5.An example of $\mu N P D t e s t$ run for the three analines are shown in Figure 6. Aciddissociation constants $\left(\mathrm{pK}_{\mathrm{a}}\right)$ for the amino group on the anilines are given alongside the structure of each amine shown in Figure 4 and in Figure 5. $\mathrm{pK}_{\mathrm{a}}$ values were observed in order to give an estimation of electron density surrounding the $\mathrm{N}$ atom. A pKa value is an estimation of the affinity for electrons on the nitrogen atom in solution. Testing the selectivity of the $\mu$ NPD determined that certain amines are detected better than others. Substituted anilines were found to be detected in a manner which was correlated with increasing $\mathrm{pK}_{\mathrm{a}}$ values of the amino group on the anilines. The $\mu$ NPD experiments were performed under $\mathrm{N}_{2}$, not in solution where $\mathrm{pK}_{\mathrm{a}}$ values are determined. A more sophisticated determination of electron density for the $\mathrm{N}$ atom in the aniline molecules is needed to better understand the correlation of electron density and $\mu$ NPD response.

For nitrogen containing molecules, proton elimination at the amino group is energetically favorable in the gas phase [10]. This gives evidence that at high temperatures, anilines and anilines with substituents are more easily able to be ionized due to loss of a proton on the amino group causing a response on the $\mu$ NPD. This indicates that structure based alterations in the amino group need to be observed. Higher $\mathrm{pK}_{\mathrm{a}}$ values for the amino group in anilines indicate that there is a higher electron density surrounding the nitrogen. Analysis of $\mathrm{pK}_{\mathrm{a}}$ values and various atomic charge schemes show strong correlations between $\mathrm{pK}_{\mathrm{a}}$ and atoms-in-molecules (AIM) charge, Löwdin charge, and natural charge [11]. AIM charge predicts that an increase in positive charge on the $\mathrm{N}$ atom allows for a better acceptance of 
electrons. Contradictory to the AIM charge, both Löwdin and natural charge predict that higher electron density (negative charge) on the $\mathrm{N}$ atom correlate with a greater peak area. For the $\mu$ NPD, it is proposed that electrons are emitted from the alkali metal ( $\mathrm{Cs}$ or $\mathrm{Rb}$ ) atoms which supply the $\mathrm{N}$ atom with electrons. The additional electrons give the sample a negative charge which is proportional to the basicity of the nitrogen atom on the molecule. Thermionic emission of the $\mu$ NPD determines that a higher electron density surrounding an atom with a large ionization cross section area correlates to a larger $\mu$ NPD peak area. $\mathrm{N}$ and $\mathrm{P}$ atoms have a large ionization cross section area due to their molecular orbital structure. In their base state $\mathrm{N}$ atoms and $\mathrm{P}$ atoms have five electrons surrounding them, while two of these electrons constitute a lone pair for anilines. This large electron cloud surrounding both $\mathrm{N}$ and $\mathrm{P}$ atoms is much larger than for atoms such as $\mathrm{C}$. This explains why $\mathrm{N}$ and $\mathrm{P}$ containing molecules are detected better than hydrocarbons in the case of commercial NPDs $\left(7^{*} 10^{4} x, 10^{5} x[2]\right)$.

In order to further test this theory, determination of electron density on the $\mathrm{N}$ atom of $\mathrm{N}$ containing molecules needs to be calculated. Correlation of calculated electron density and $\mu$ NPD selectivity could be further examined. This analysis would need to be considerate of the heated surface containing a $\mathrm{CsOH}$ or $\mathrm{RbOH}$ thin film and the possibility of both a gas phase mechanism and a surface ionization mechanism for transfer of electrons.

Using $\mathrm{N}_{2}$ as a carrier gas minimizes the formation of decomposition products due to the presence of oxygen. The sample requires electronegative functional groups in order for the thermionic source to extract electrons. The results presented here correlate with the theory that the $\mu$ NPD has a selective response based on a sample's molecular structure. The presence and location of electronegative groups on the molecular structure alter the detector's response. Further experimentation needs to be performed on molecules with varying electronegativity and electron density surrounding the $\mathrm{N}$ atom in molecules before exact mechanisms can be determined.

In order to determine the effectiveness of the $\mu$ NPD compared tothe commonly used FID, the limit of detection(LOD) was calculated for $p$-methoxyaniline and compared to 1,3,5-

trimethylbenzene.The results are shown in Table 1, and chemical structures are shown in Figure 7. The LOD for p-methoxyaniline was calculated to be $.29 \mathrm{ng}$ for the $\mu$ NPD at a temperature of $516^{\circ} \mathrm{C}$, which is a factor of 4 better than the LOD of 1.16ng for the Flame Ionization Detector.

\section{Conclusions}

Selectivity of the $\mu$ NPD was tested for three amines: aniline, $\mathrm{p}$-fluoroaniline, and $\mathrm{p}$ methoxyaniline on a $\mathrm{CsOH}$ coated device. Amines were found to be detected in a manner correlating with $\mathrm{pK}_{\mathrm{a}}$ values: $\mathrm{p}$-methoxyaniline $>\mathrm{p}$-fluoroaniline $>$ aniline. Sensitivity of the $\mu$ NPD for $\mathrm{p}$-methoxyaniline was found to be $0.29 \mathrm{ng}$ on a $\mathrm{RbOH}$ coated device which is an improvement in detection compared to the FID. The $\mu$ NPD discussed here could be improved upon by various methods of increasing electrical shielding, improving coating pre/post treatment, and improving $\mu$ NPD device design to increase longevity and durability to allow for higher temperatures and longer run times. Size and sensitivity of the $\mu$ NPDis promising for incorporation of the $\mu$ NPD in a portable chemical detection system. 


\section{References}

1. Bombick, D.D. and J. Allison, Investigations Into the Response Mechanism of the Gas Chromatographic Thermionic lonization Detector Part I. Mass Spectral Studies. Journal of Chromatographic Science, 1989. 27(10): p. 612-619.

2. Patterson, P.L., Selective responses of a flameless thermionic detector. Journal of Chromatography A, 1978. 167(0): p. 381-397.

3. Carlsson, H., G. Robertsson, and A. Colmsjo, Response mechanisms of thermionic detectors with enhanced nitrogen selectivity. Anal Chem, 2001. 73(23): p. 5698-703.

4. Draper, W.M., Optimizing nitrogen-phosphorus detector gas chromatography for pesticide analysis. Journal of Agricultural and Food Chemistry, 1995. 43(8): p. 2077-2082.

5. Chattopadhyay, K. and A. Banerjee, Introduction To Nanoscience And Nenotechnology. 2009: PHI Learning Pvt. Ltd.

6. Erickson, B., Product Review: Measuring nitrogen and phosphorus in the presence of hydrocarbons. Analytical Chemistry, 1998. 70(17): p. 599A-602A.

7. Simonson, R.J., et al., Microfabricated nitrogen-phosphorus detector: chemically mediated thermionic emission, 2012, Sandia National Laboratories.

8. Oblinger, C.J., et al., New reporting procedures based on long-term method detection levels and some considerations for interpretations of water-quality data provided by the US Geological Survey National Water Quality Laboratory. 1999: p. 3.

9. López, R., et al., Determination of minor and trace volatile compounds in wine by solid-phase extraction and gas chromatography with mass spectrometric detection. Journal of Chromatography A, 2002. 966(1-2): p. 167-177.

10. Pankratov, A.N., et al., Correlations between the Basicity and Proton Affinity of Substituted Anilines. Journal of Structural Chemistry, 2001. 42(5): p. 739-746.

11. Gross, K.C., P.G. Seybold, and C.M. Hadad, Comparison of different atomic charge schemes for predicting $p K a$ variations in substituted anilines and phenols*. International Journal of Quantum Chemistry, 2002. 90(1): p. 445-458.

12. Gross, K.C. and P.G. Seybold, Substituent effects on the physical properties and pKa of aniline. International Journal of Quantum Chemistry, 2000. 80(4-5): p. 1107-1115. 


\section{Figure and Table Captions}

Table 1: A RbOHcoated $\mu N P D$ device is tested and compared to FID data for $p$-methoxyaniline (best detected out of three anilines tested) and compared to an all carbon molecule of similar molecular weight. $\mu N P D$ is at $516^{\circ} \mathrm{C}$

Figure 1: Uncoated $\mu N P D$ (microhotplate) device next to a dime.

Figure 2: $\mu N P D$ testing setup- SMA connectors, Macor ${ }^{\circledR}$ inserts (in white), and stainless steel fixture, the $\mu N P D$ fits in between two Macor ${ }^{\circledR}$ inserts[7].

Figure 3:Schematic of the electrical connections and gas flow used in the testing setup. Several external power supplies are used to apply the proper biases to the testfixture, $\mu N P D$ device, and collector electrode. A Keithley Model 6514 Electrometer reads the charge collectedfrom the sensor.The electrometer is biased to a positive potential, using an external power supply, relative to thefixture walls which are held at system ground. The $\mu N P D$ (microhotplate) is biased negatively to thefixture and the collector electrode using an additional power supply. This creates a large voltagewithin the fixture that drives negative charge from the emitter film on the $\mu N P D$ device to thecollector electrode above it. The large voltage within the fixture drives electrons from the emitter film on the $\mu N P D$ device to the collector electrode above it.

Figure 4: Selectivity of amines, determined by peak area ( $\left.p A^{*} s\right)$ for each analyte, tested on a $\mathrm{CsOH}$ coated $\mu N P D$ device. Acid dissociation constant $\left(p K_{a}\right)$ values for the amino group on the anilines are in parentheses [12].

Figure 5:Relative sensitivity of $\mathrm{CsOH} \mu N P D$ to anilines. Peak areas ranging from largest to smallest: $p$ methoxyaniline, $p$-fluoroaniline, and aniline. Acid disassociation constants $\left(p K_{a}\right)$ for the amino group on the anilines are correlated to $\mu N P D$ detection ability for this aniline series [12]. Temperature in degrees Celsius is on the $x$-axis and the ratio of peak areas of $\mu N P D$ : FID (microfabricated nitrogen phosphorus detector: flame ionization detector) is on the $y$-axis. Error bars show one standard deviation.

Figure 6: Aniline series tested on $\mathrm{CsOH} \mu N P D$. Samples are at a concentration of $0.1 \mathrm{M}$ in acetonitrile. The solvent peak for acetonitrile is on the left. $\mu N P D$ is at $463^{\circ} \mathrm{C}$.

Figure 7 (right): Chemical structure of p-methoxyaniline and 1,3,5-trimethybenzene. 
Table and Figures

Table 1-Amine Compared to Carbon Compound

\begin{tabular}{|c|c|c|}
\hline \multirow{4}{*}{$\begin{array}{l}\text { p-Methoxyaniline } \\
\text { 1,3,5-Trimethylbenzene }\end{array}$} & \multicolumn{2}{|c|}{ Signal to Noise Ratio } \\
\hline & FID & $\mu \mathrm{NPD}$ \\
\hline & 0.91 & 3.7 \\
\hline & 3.6 & 4.6 \\
\hline & \multicolumn{2}{|c|}{ Limit of Detection (ng) } \\
\hline & FID & $\mu \mathrm{NPD}$ \\
\hline p-Methoxyaniline & 1.2 & 0.29 \\
\hline 1,3,5-Trimethylbenzene & 75 & 59 \\
\hline
\end{tabular}

Figure 1-Uncoated $\mu$ NPD Device

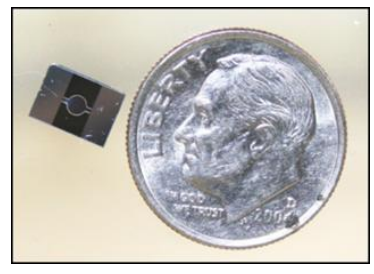

Figure 2- $\mu$ NPD Testing Setup

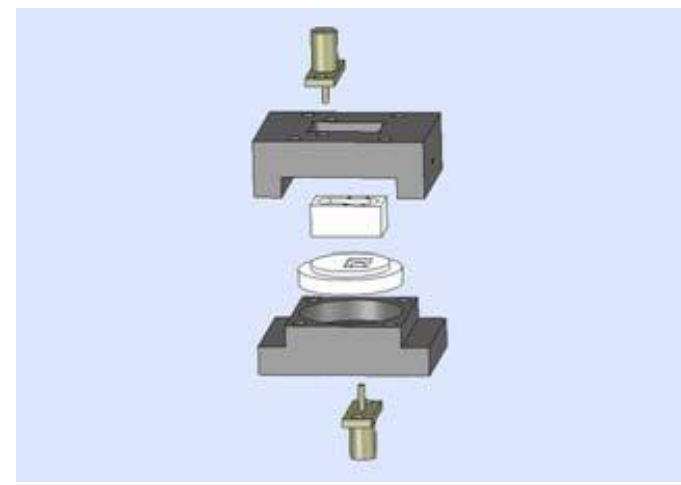


Figure 3-Schematic of Setup

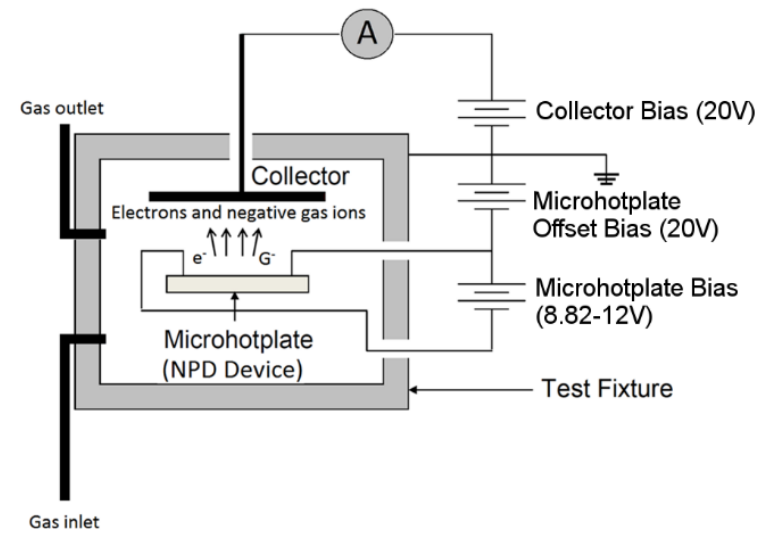

Figure 4-Selectivity of Amines<smiles>COc1ccc(N)cc1</smiles>

Figure 5- $\mu$ NPD Sensitivity

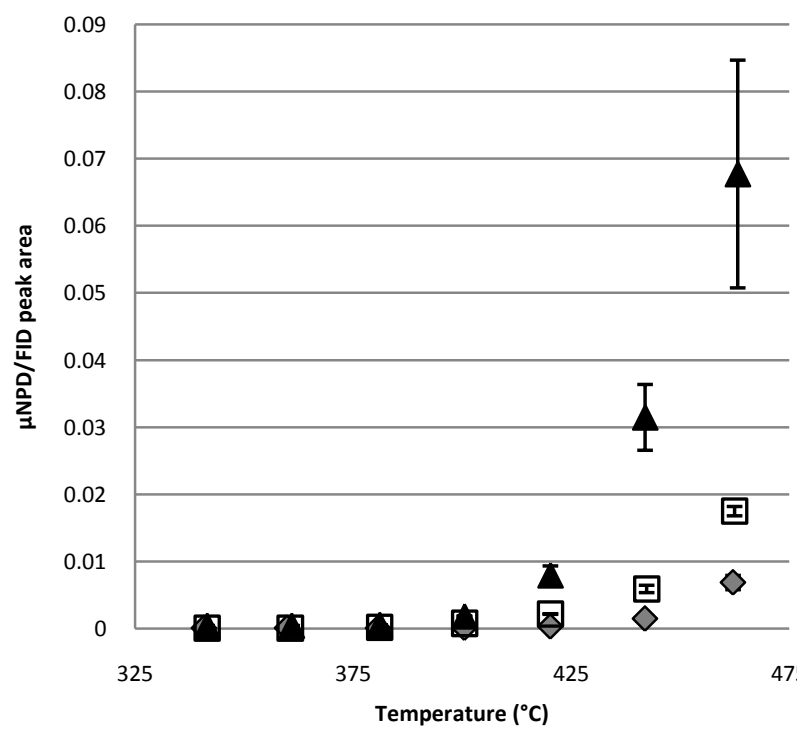

$\diamond$ Aniline (pKa 4.58)

口p-fluoroaniline (pKa 4.65electron withdrawing)

$\Delta$ p-methoxyaniline (pKa 5.29electron donating) 
Figure 6-Aniline Series Comparison

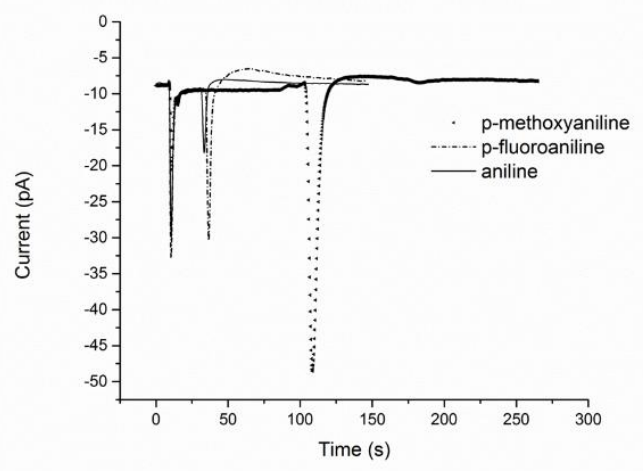

Figure 7- Amine and Carbon Compound Chemical Structures

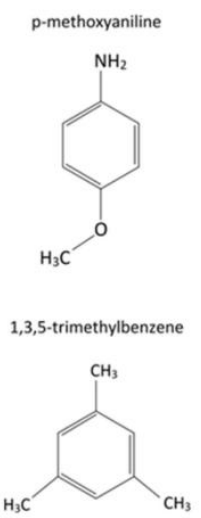

